\title{
All-optical self-referenced transverse position sensing with sub-nanometer precision
}

\author{
Nora Tischler, ${ }^{\dagger} \#$ Johannes Stark, ${ }^{\ddagger}, \#$ Xavier Zambrana-Puyalto, "Ivan \\ Fernandez-Corbaton, ${ }^{\S}$ Xavier Vidal, ${ }^{\ddagger}$ Gabriel Molina-Terriza, ${ }^{\prime}$ and Mathieu L. \\ Juan $^{*, \perp}$ \\ †Centre for Quantum Dynamics, Griffith University, Brisbane 4111, Australia \\ $\ddagger$ Department of Physics \& Astronomy, Macquarie University, NSW 2109, Australia and \\ ARC Centre for Engineered Quantum Systems, Macquarie University, NSW 2109, Australia \\ ฯIstituto Italiano di Tecnologia, Via Morego 30, 16136 Genova, Italy \\ $\S$ Kalsruhe Institute of Technology, Kalsruhe, Baden-Wuerttemberg, Germany \\ \|Centro de Física de Materiales (MPC) and Donostia International Physics Center \\ (DIPC), 20018 Donostia-San Sebastián, Spain and \\ IKERBASQUE, Basque Foundation for Science, 48013 Bilbao, Spain \\ $\perp$ Institute for Quantum Optics and Quantum Information of the Austrian Academy of \\ Sciences, A-6020 Innsbruck, Austria \\ \# These authors contributed equally to this work \\ E-mail: mathieu.juan@uibk.ac.at
}

\begin{abstract}
The emergence of technologies operating at the nanometer scale for applications as varied as nano-fabrication and super-resolution microscopy has driven the need for ever more accurate spatial localization. In this context, nano-structures have been used as
\end{abstract}


probes in order to provide a reference to track lateral drifts in the system. Yet nanometer precision remains challenging and usually involves complicated measurement apparatus. In this work we report a simple method based on symmetry considerations to measure the position of a sub-wavelength nano-structure. For a particular choice of structures, gold nano-particles, we demonstrate a sub-nanometer lateral precision of $0.55 \mathrm{~nm}$. The versatility of the method also allows for the use of different structures, offering a promising opportunity for sub-nanometer positioning accuracy for a wide variety of systems.

Keywords: position sensing, angular momentum, nano-particle, helicity, symmetry breaking

In both research and industry, the decreasing size of components along with the increasing technological complexity drive the demand for positioning samples with sub-nanometer precision. To mention only a few examples, in the semiconductor industry the emergent technology of monolithic 3D architectures promises better scaling than the current 2D Moore scaling, which is already showing signs of diminishing returns. ${ }^{1}$ Yet, this approach requires much higher precision for the positioning of wafers, in particular for the bonding step. ${ }^{2}$ In parallel, the field of optical microscopy has also been pushing the limits of optical imaging to the nanometer scale with the advent of super-resolution microscopy. ${ }^{3,4}$ Operating at resolutions of the order of a few nanometers requires both accurate positioning and stabilization of the sample. In particular, methods relying on the use of multiple images with long acquisition time, such as PALM ${ }^{5}$ or STORM, ${ }^{6}$ are very sensitive to sample drift.

As a consequence, a large variety of methods has been explored in order to accurately measure the position of a sample with different constraints depending on the specific application. Diffraction Based Overlay (DBO) is a typical all-optical positioning method used in the semiconductor industry for the bonding process, providing sub-nanometer accuracies. ${ }^{7}$ For this technology, the grating quality and, most importantly, its size are critical to maintain sub-nanometer performances. On the other hand, the number of final devices per wafer 
constitutes a strong constraint in the industry, and reducing the size of DBO gratings represents an active area of research. Recent efforts have allowed the development of $\mu \mathrm{DBO}$, but regions of the order of $10 \mu \mathrm{m}^{2}$ are still required. ${ }^{8}$ In the field of optical microscopy, nano-scale structures such as fluorescent probes or metallic nanoparticles have been widely employed as sample position markers. Using methods that rely on determining the centroid of the pointspread function, lateral resolutions of $10 \mathrm{~nm}$ and better have been demonstrated, ${ }^{9}$ allowing for single fluorophore imaging with $1.5 \mathrm{~nm}$ localization. ${ }^{10}$ In general, centroid-based methods are limited to precisions down to a few nanometers even with recent algorithms ${ }^{11,12}$. In the context of dSTORM microscopy ${ }^{13}$ active sample stabilization was successfully implemented using gold nanoparticles, increasing the resolution by $26 \% .{ }^{14}$ By measuring both the phase and amplitude of the field, this method provides more information from the scattered field and leads to a lateral localisation precision of $1.5 \mathrm{~nm}$ when using a single gold nanosphere. Alternatively, methods relying on only measuring the amplitude can be improved by using optical nanoantennas which exhibit strongly directional light scattering, where a small displacement of the nanoanteanna can produce a strong variation of the scattered field. ${ }^{15}$ While these methods have reached sub-nanometer lateral resolutions, the fact that they either require complex apparatus (e.g. phase and amplitude imaging) or intricate structures (e.g. resonant nanoantennas or diffraction gratings) significantly limits their versatility.

In this work, we propose a novel method based on general considerations related to the symmetries of the system. The concept can be described as follows: using a scattering structure and an incident field that both present a cylindrical symmetry with identical axis directions, the whole system is cylindrically symmetric only when the scatterer is centered with respect to the incident beam. By accurately monitoring the pattern of the scattered field, and in particular its asymmetry, the displacement of the scatterer from the beam center can be inferred. This effect can be viewed in terms of angular momentum conservation, related to the cylindrical symmetry: as the scatterer is moved off-center, additional components of the angular momentum are allowed, consequently modifying the scattered field 

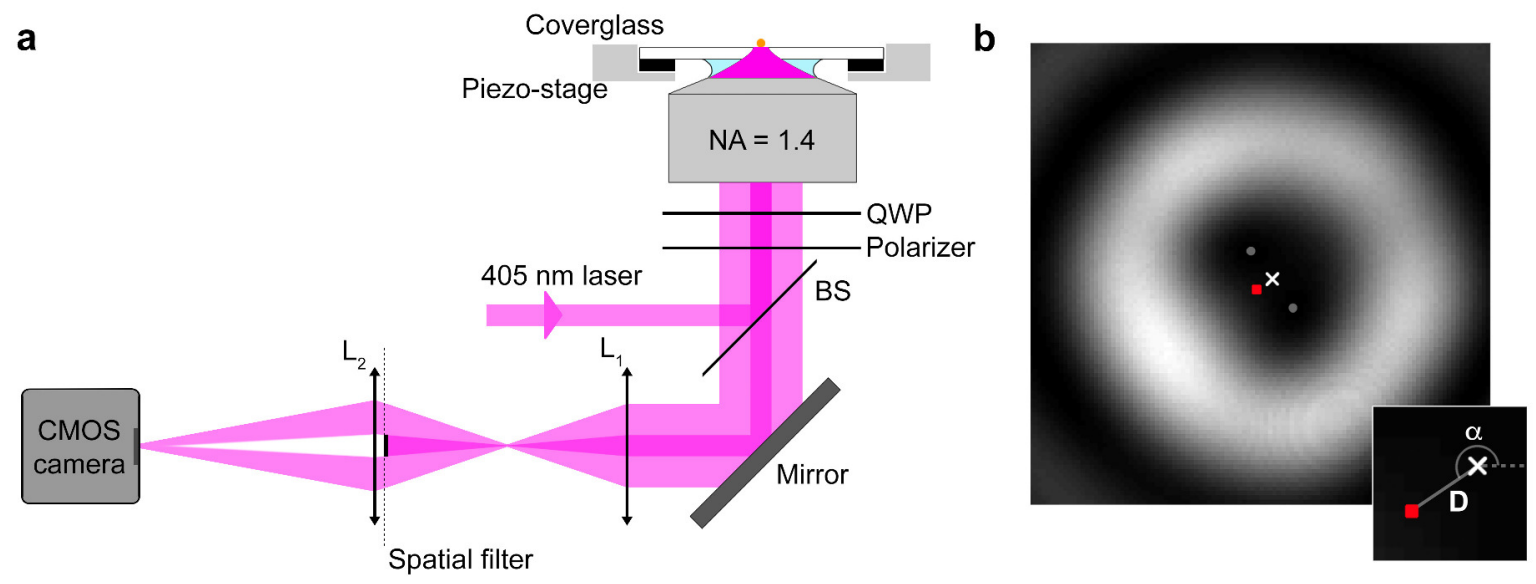

Figure 1: Experimental approach. a, the experimental setup is based on an inverted microscope with a piezo-stage to control the position of the sample. A $405 \mathrm{~nm}$ laser is focused using a 1.4 NA objective, and its polarization is controlled using a polarizer and a quarter-waveplate (QWP). The backscattered light is collected through the same objective and the set of QWP/polarizer allows to isolate the polarization component producing the doughnut beam. After the beam-splitter (BS), the lens $\left(\mathrm{L}_{1}\right)$ allows to conjugate the Fourier plane (objective rear aperture) to the spatial filter. The resulting dark-field image is produced on the complementary metal-oxide semiconductor (CMOS) camera with a lens doublet $\mathrm{L}_{2}$. $\mathbf{b}$, the image processing of the scattered field pattern. The magnitude $D$ and angle $\alpha$ (see inset) are obtained from the midpoint (white cross) between the two intensity minima related to the two optical vortices (gray solid circles), and the intensity weighted centroid (red solid square). 
pattern. This method is amenable to a study using general mathematical principles, showing that even the condition of cylindrical symmetry can be relaxed ${ }^{18}$ and small deviations from the ideal system will not affect the measurement. For these reasons, the approach presents several advantages with no constraints on the scatterer nature or the incident beam besides their geometrical symmetry, and a simple measurement apparatus relying on the scattered field intensity imaging. In this letter, we focus on a particular implementation of this approach using gold nanospheres probed by a circularly polarized Gaussian beam and demonstrate a lateral localization of $0.55 \mathrm{~nm}$. In addition, we show that the gold nanospheres can be used as a reference point to compensate for various sources of drift of the experiment when the stage is quickly driven to different positions. To emphasize the versatility of the approach we also present results obtained with a different type of nanostructure, namely circular nanoapertures in a gold film.
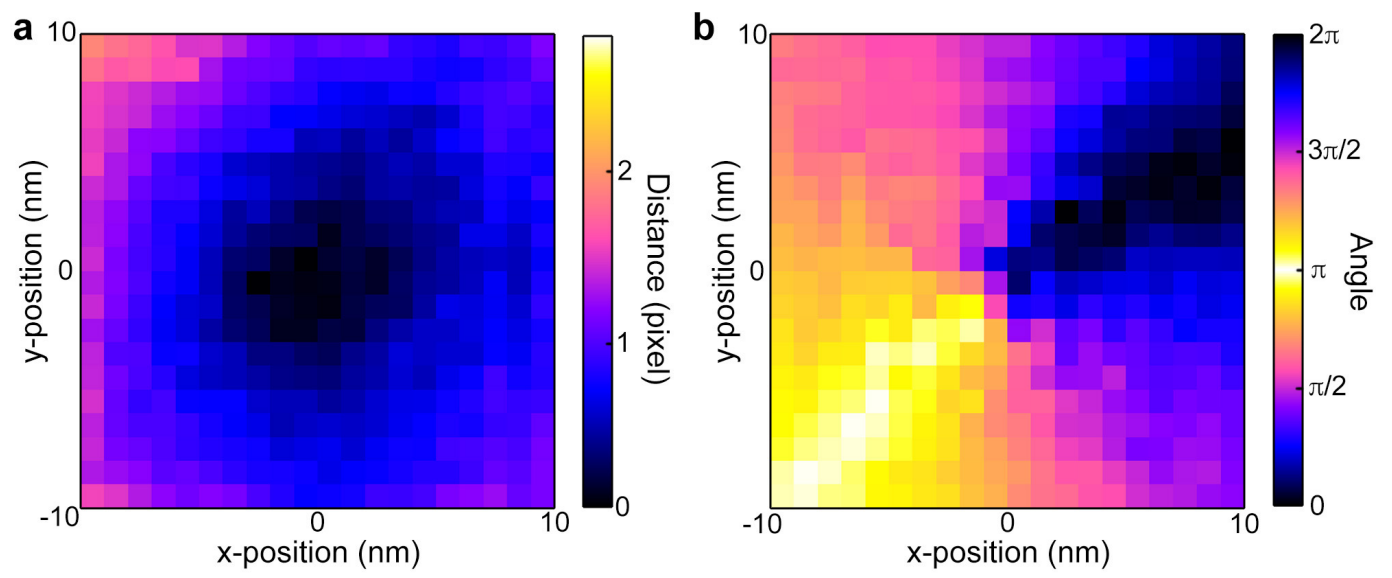

Figure 2: Experimental 2D scans. The scan extends over a region of $20 \times 20 \mathrm{~nm}^{2}$ with a step of $1 \mathrm{~nm}$. The results from the image processing are presented in terms of a, magnitude $D$ and $\mathbf{b}$, angle $\alpha$.

The experimental setup, depicted in Fig. 1a, consists of a left circularly polarized Gaussian beam focused with a high numerical aperture objective $(\mathrm{NA}=1.4)$ onto a single $125 \mathrm{~nm}$ diameter gold nanosphere deposited on a glass coverslip (see Supporting Information). The scattered field is back collected through the same objective and spatially filtered such that the strong back-reflection from the coverglass is removed. Such dark-field configuration is 
achieved by under-filling the objective and filtering out these components in the imaging part via a spatial filter (see Fig. 1a). To further improve the precision of our method, only the crossed-polarized component of the backscattered field (right circularly polarized) is imaged on a digital camera (see Fig. 1b). When the gold nanosphere is centered with the incident beam, the symmetry of the whole system imposes conservation of the total angular momentum $\left(J_{z}=1\right)$ while due to the lack of electromagnetic duality symmetry of the system, the helicity (or circular polarization for collimated beams) can change through the scattering process. ${ }^{16}$ As a consequence, the scattered far-field contains both helicities $(\Lambda= \pm 1)$ where one helicity component presents a Gaussian distribution and the other ideally presents an optical vortex of charge 2. By imaging only the latter, the particular pattern of this doughnut beam facilitates tracking its distortion as a function of the gold nanosphere position relative to the incident beam. Due to the imperfect polarization selection in the experiment and the inherent instability of the charge 2 optical vortices, the pattern presents instead two optical vortices of charge $1 .{ }^{17}$ To characterise the doughnut beam distortion, the image processing algorithm retrieves two particular points from the scattered field pattern: the first point defined as the midpoint between the two local minima of the field related to the two optical vortices, and the second point defined as the intensity weighted centroid of the pattern. From these two points, we define a vector in the camera reference frame with magnitude $D$ and angle $\alpha$ (see Fig. 1b inset). Other points in the image can alternatively be chosen to acquire similar information, ${ }^{18}$ but our choice of points along with the processing algorithm provides good stability and sensitivity (see Supporting Information). When the gold nanosphere is centered, the pattern is expected to present a very high degree of symmetry, only limited by experimental inaccuracies, so that the distance between the points obtained (vector magnitude $D$ ) will then be minimized and approaching 0. Conversely, as the nanosphere is moved away, the symmetry of the pattern will degrade rapidly, consequently increasing the vector magnitude $D$. The value of $D$ and $\alpha$ can be interpreted as the magnitude and the direction of the pattern distortion, respectively. 

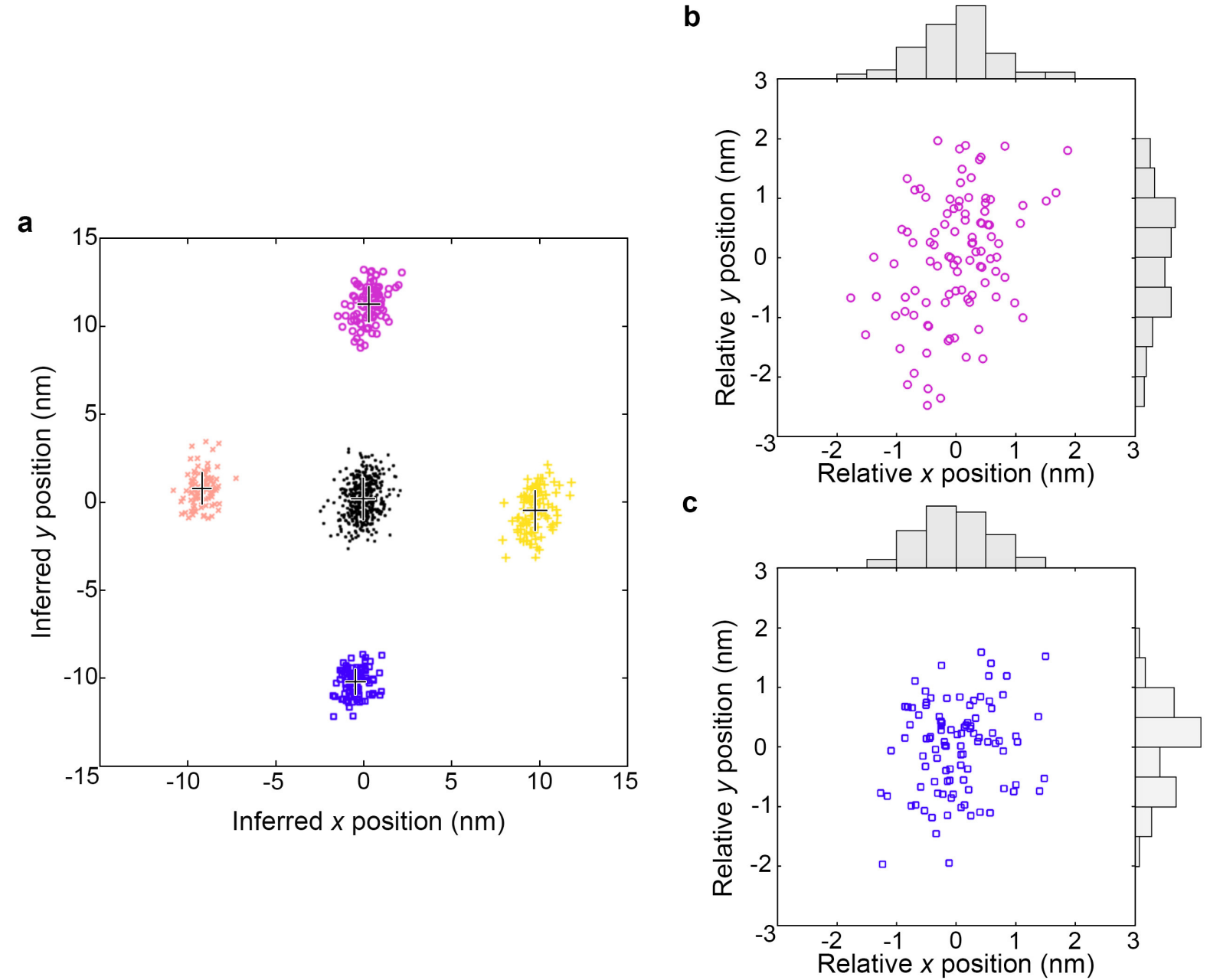

Figure 3: Drift correction. a, the sample is moved between 4 different extremum positions $(x= \pm 10 \mathrm{~nm}$ and $y= \pm 10 \mathrm{~nm}$ ) returning to the central position each time. Any offset between the central position and the reference position, defined as $D=0$, is corrected for, allowing the compensation for any potential drift occurring in the system along with cumulative errors from the piezo-stage (stage precision and hysteresis). $\mathbf{b}$ and $\mathbf{c}$ present the detail of the positions ( $x=0 \mathrm{~nm}, y=10 \mathrm{~nm})$ and $(x=0 \mathrm{~nm}, y=-10 \mathrm{~nm})$, respectively, along with the position histograms along the $x$ - and $y$-directions. For clarity, the inferred positions are re-centered. 
We now demonstrate the possibility to define a reference position as the position of the gold nanosphere that minimizes the magnitude $D$. This is achieved by moving the sample in steps of $1 \mathrm{~nm}$ using a piezo nanopositioner to obtain a 2D scan of the region around the expected reference position. For each stage position, and to reduce the effect of mechanical drift, 50 images are taken in rapid succession by using short exposure times (1 ms) and a small active area for the CMOS sensor $(144 \times 144$ pixels $)$. These images are averaged in groups of 5 successive images in order to reduce noise and increase the dynamic range and the resulting 10 averaged images are processed in order to obtain the values of $D$ and $\alpha$. Figure 2 presents the 2D maps for both the magnitude $D$ and the angle $\alpha$ for a $20 \times 20 \mathrm{~nm}^{2}$ region (1 nm steps). As expected, the magnitude $D$ changes slowly with the displacement of the nanosphere when we are considering such a small range of motion. Remarkably, the angle $\alpha$ presents a singularity which corresponds to the sample position where the magnitude $D$ is 0 and the angle cannot be defined. This singularity is a topological feature of the measurement space and is consequently expected to be stable even under continuous deformation of the system. ${ }^{18}$ For example small deformations or imperfections of the scattering object or the incident beam will still provide a singularity in the measurement space, although the precision might be impacted depending on the degree of deformation. In the case of subwavelength particles, the asymmetry of the particle will not be relevant for wavelengths at which the particle effectively behaves as a point dipole. On a practical level, this singularity can be used to define a reference position. When the step size used is comparable or larger than the precision of the method, the 2D maps show little noise and the reference position is then defined to within 1 pixel. For example, for the maps presented in Fig 2 we can identify the reference position with a precision related to the step size of $1 \mathrm{~nm}$. We note that due to the large number of experimental measurements contained in the $2 \mathrm{D}$ maps, the use of a simple fitting function would allow to determine the reference position with a much higher precision (see Supporting Information). In addition, for an off-center position of the sample, obtaining the value for $D$ and $\alpha$ is sufficient to know the position of the sample relative 
to the reference, assuming prior knowledge of the full $2 \mathrm{D}$ map in the region considered. In order to retrieve a calibration from the 2D maps, Cartesian coordinates are obtained from the values of $D$ and $\alpha: x_{p x}=D \cos (\alpha)$ and $y_{p x}=D \sin (\alpha)$ where the units are in terms of the camera pixels. The calibration, assuming a linear dependence for a sufficiently small range of positions, can simply be expressed in matrix form as:

$$
\left(\begin{array}{l}
x_{p x} \\
y_{p x}
\end{array}\right)=\left(\begin{array}{ll}
a & b \\
c & d
\end{array}\right)\left(\begin{array}{l}
x \\
y
\end{array}\right)
$$

where $\left(\begin{array}{l}x \\ y\end{array}\right)$ is the physical position of the piezo-stage. The calibration matrix is then inverted such that for a subsequent measurement the physical position can be inferred from $\left(\begin{array}{l}x_{p x} \\ y_{p x}\end{array}\right)$. Asymmetry of the 2D maps, visible from the elliptical shape of the distance $D$, will also propagate to the calibration matrix. This asymmetry likely originates from the beam preparation and focusing (e.g. imperfect polarization control and astigmatism).

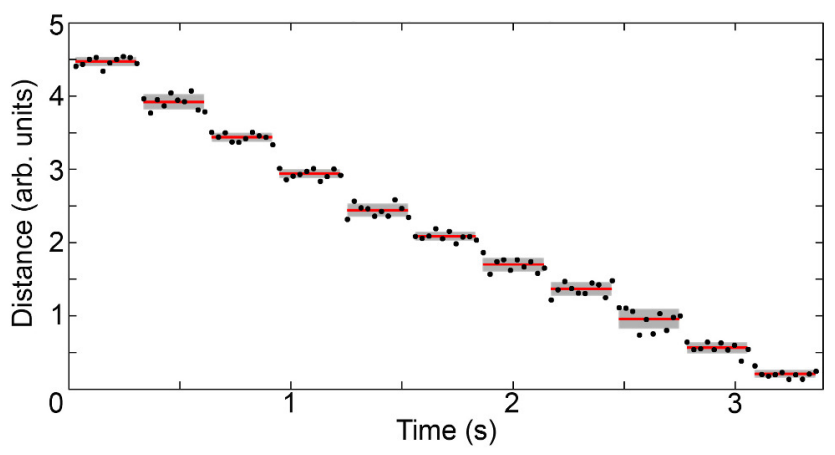

Figure 4: Precision. $30 \mathrm{~nm}$ line scan along the $x$-direction toward the reference position with a $3 \mathrm{~nm}$ step. For each stage position 50 images were taken and averaged in groups of five, the resulting 10 averaged images were later processed in order to obtain the values of $D$. The mean value of the magnitude is calculated for each step (solid line), along with the 1- $\sigma$ interval (shaded region).

Taking advantage of the reference position, we demonstrate an active feedback approach that can be used to stabilize the position of the sample even when the stage is frequently moved. After obtaining the 2D maps we moved the sample to 4 different positions: $x=$ $\pm 10 \mathrm{~nm}$ and $y= \pm 10 \mathrm{~nm}$. Between each extremum position, the sample is re-centered and 
any offset to the reference position $(D=0)$ is corrected for. This active feedback allows to correct for any drift in the experiment but also for cumulative errors due to the piezo-stage hysteresis. Each cycle, defined by moving the stage to an extremum position and then back to the center for each of the 4 different extremum positions, with a total of 8 stage positions per cycle was repeated 100 times. For each of the 800 positions, 50 images are taken and averaged in groups of five; the resulting 10 averaged images are processed in order to obtain the values of $D$ and $\alpha$. By using the calibration from the $2 \mathrm{D}$ maps the $x$ and $y$-positions are inferred, displayed in Fig. 3, showing an average standard deviation of $0.65 \mathrm{~nm}$ and $1 \mathrm{~nm}$ in the $x$ and $y$-direction, respectively. Conversely, in the camera frame $\left(\begin{array}{l}x_{p x} \\ y_{p x}\end{array}\right)$ the standard deviation is isotropic (see Supporting Information). Noise originating from the image processing, such as image shot noise, would lead to an isotropic error in the camera frame. In this case, any asymmetry from the 2D maps and consequently the calibration will then be directly imparted on the inferred $\left(\begin{array}{l}x \\ y\end{array}\right)$ position. This is consistent with our experimental observation, suggesting that the precision is mostly limited by noise in the imaging system. We also note that by correcting for the central position to coincide with the reference position, the cumulated offset correction that was applied in this case reached $4.5 \mathrm{~nm}$. For the drift correction the image acquisition time amounts to $0.1 \mathrm{~s}$ and the image processing time to $1.2 \mathrm{~s}$ on average, making this approach particularly adapted to correct for slow drifts, for example drifts related to temperature changes. Our method combined with the piezoelectric feedback allows for compensating both slow drifts and fast mechanical instabilities, achieving a positioning accuracy difficult to obtain otherwise.

In order to better assess the precision of the method, we also performed a $30 \mathrm{~nm}$ line scan along the $x$-direction towards the centre with a $3 \mathrm{~nm}$ step width. By using only 10 steps, from $x=30 \mathrm{~nm}$ to $x=0 \mathrm{~nm}$, the cumulative error from the piezo-stage hysteresis is expected to be minimal. The images were not processed in real-time in this case, so that the scan was obtained in the shortest possible time to reduce the impact of drift in the system. As previously done, for each stage position, 50 images were taken and averaged in groups of 
five, the resulting 10 averaged images were later processed in order to obtain the values of $D$ and $\alpha$. The result from the line scan, in terms of the magnitude $D$, is presented in Fig. 4 where each step is well resolved. The calibration obtained from 2D scans was not used in this case in order to minimize the error related to the linear approximation of such a calibration. Instead, knowing each step is nominally $3 \mathrm{~nm}$, an effective calibration is obtained by relating the difference between the average values of $D$ before and after the step to a $3 \mathrm{~nm}$ position change. This enabled us to express the standard deviation at each stage position in nanometers, leading to an average standard deviation of $0.55 \mathrm{~nm}$ for this line scan. This calibration leads to an uncertainty associated with the lateral precision of the piezo-stage, and using standard error propagation, we obtained a negligible error of $0.01 \mathrm{~nm}$ for this uncertainty (see Supporting Information). It is important to note that the 1- $\sigma$ precision we have measured, $0.55 \mathrm{~nm}$, is related to short term mechanical instabilities in the system (e.g. vibrations), the stage active feedback accuracy $(0.3 \mathrm{~nm})$ and the precision of our measurement method. Assuming instead a perfect point-dipole scatterer and point-spread function, no background noise and a perfect compensation from the piezo feedback of all mechanical instabilities, we obtain a theoretical lower bound for the precision of centroid-based localization of $0.47 \mathrm{~nm}$ (see Supporting Information). Our method provides a localization precision only $17 \%$ higher than best case estimate of the theoretical bound for centroid-based method. Moreover, by using the symmetry of the pattern, the localization information is related to the relative position of the scatterer with regard to the incident beam. As a consequence, potential drifts occurring in the imaging part of the experiment do not impact the precision, providing an important advantage over conventional position measurement methods such as centroid-based algorithms and split-detectors sensing. ${ }^{19}$ Indeed, while these methods can reach nanometer-scale precisions, they are not suitable to provide a robust reference position. This also impacts the design of the experiment where, for our method, greater care was given to achieving high stability for the incident beam (see Supporting Information).

Remarkably, our method does not rely on specific materials or resonances, but instead 


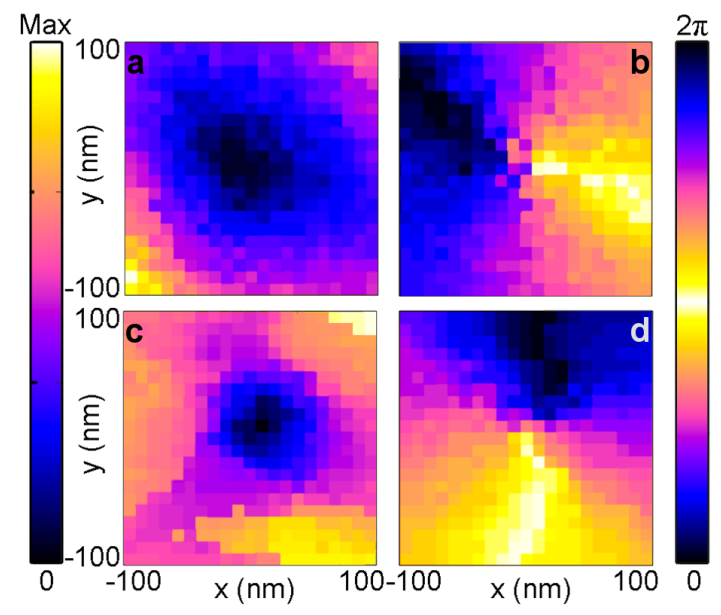

Figure 5: Reference position for a nanoaperture. The scans extend over an area of $200 \times 200 \mathrm{~nm}^{2}$, with a step of $10 \mathrm{~nm}$. The top and bottom rows correspond to results for nanoapertures with diameters of $500 \mathrm{~nm}$ and $630 \mathrm{~nm}$, respectively. a and c, magnitude $D$. b and $\mathbf{d}$, angle $\alpha$.

relies on some geometrical considerations of the whole setup. As a consequence, a wide variety of structures could be used in a similar way, along with virtually any wavelengths. To demonstrate this important versatility, we performed 2D scans using a completely different structure and setup: an isolated circular nanoaperture in a $150 \mathrm{~nm}$ thick gold film measured in transmission. Excited with a circularly polarized beam, the scattered field was collected in transmission and selected in polarization to isolate the doughnut beam. Although the mechanical stability of this experimental setup did not allow for sub-nanometer precision (1- $\sigma$ precision of $4.5 \mathrm{~nm})$, the reference position $(D=0)$ was retrieved for different aperture sizes, as shown in Fig. 5. Similarly to the nanoparticle the retrieved scan maps for both nanoapertures depart from the expected cylindrical symmetry. We also note that while both $120 \mathrm{~nm}$ colloidal particles and circular nanoapertures did not present perfect a cylindrical symmetry, the singularity was always present in the measurement space. The expected larger asymmetry for the nanoapertures compared to the colloidal particles could have contributed to the lower precision of $4.5 \mathrm{~nm}$ for the nanoapertures.

In conclusion, in this letter we have proposed a novel method based on symmetry considerations that allows for sub-nanometer lateral localization precision. For a scatterer and an 
incident field both presenting cylindrical symmetry along the same axis direction, a reference position can be determined when the scatterer is centered with regard to the incident beam. Through the analysis of the scattered field intensity, the offset from such reference position was obtained with a lateral precision of $0.55 \mathrm{~nm}$ using a $125 \mathrm{~nm}$ gold nanosphere as the scatterer. This approach provides a higher precision than recent experimental centroid-based methods ${ }^{11,12}$ and access to a reference position that is independent of the exact position of the imaging system. In addition, the versatility of the method makes it suitable for a large variety of structures along with virtually no constraints on the wavelength away form resonances. The presence of strong resonances can impact this method in different ways by either increasing or completely preventing cross-polarized scattering in the case of anti-dual and dual particle respectively. ${ }^{20,21}$ For subwavelength objects such as nanorods, resonances can also present a very strong spatial asymmetry that will impact the cylindrical symmetry of the system and consequently the precision of this method. An interesting future direction for nanoparticles presenting a complex shape could be to combine our method with shape characterization. ${ }^{22}$ With the recent progress in super-resolution microscopy ${ }^{23}$ imposing growing constraints on the stabilisation of the sample, our approach would offer a robust reference position compatible with nanometer-scale resolutions, and minimal modifications to current super-resolution systems. In addition, for footprint sensitive applications such as industrial fabrication processes, the reference structure can be deeply sub-wavelength. By combining simple optical elements and sub-nanometer precision, we believe our method could be easily applied in a wide range of applications both in an industrial and a research context.

\section{Supporting information}

Additional information concerning the experimental setup, image analysis, the calibration uncertainty, the centroid-based localization uncertainty and the impact of background noise is available in the supporting information. 


\section{Acknowledgements}

This work was done in the laboratories of the Department of Physics and Astronomy (Macquarie University) and was funded by the Australian Research Council's Centre of Excellence

for Engineered Quantum Systems (EQuS). G.M.-T. also holds an Australian Research Council Future Fellowship.

\section{Competing interests}

The authors declare no competing financial interests.

\section{References}

(1) International Technology Roadmap for Semiconductors 2.0, 2015 edition, (available online).

(2) Lee, S. H; Chen, K.-N.; Lu, J. J.-Q. Wafer-to-Wafer Alignment for Three-Dimensional Integration: A Review. Journal of Microelectromechanical Systems 2011, 20, 885-898.

(3) Hell, S. W. Far-field optical nanoscopy. Science 2007, 316, 1153-1158.

(4) Huang, B.; Babcock, H.; Zhuang, X. Breaking the diffraction barrier: super-resolution imaging of cells. Cell 2010, 143, 1047-1058.

(5) Betzig, E.; Patterson, G. H.; Sougrat, R.; Lindwasser, O. W.; Olenych, S.; Bonifacino, J. S.; Davidson, M. W.; Lippincott-Schwartz, J.; Hess, H .F. Imaging intracellular fluorescent proteins at nanometer resolution. Science 2006, 313, 1642-1645.

(6) Rust, M. J.; Bates, M.; Zhuang, X. Sub-diffraction-limit imaging by stochastic optical reconstruction microscopy (storm). Nat. Methods 2006, 3, 793-796. 
(7) Blancquaert, Y.; Dezauzier, C. Diffraction based overlay and image based overlay on production flow for advanced technology node. Proc. SPIE 2013, 8681, 868104.

(8) K. Bhattacharyya, et al. On-product overlay enhancement using advanced litho-cluster control based on integrated metrology, ultra-small DBO targets and novel corrections, Proc. SPIE 8681, Metrology, Inspection, and Process Control for Microlithography XXVII, $86812 \mathrm{O}(2013)$.

(9) Thompson, R. E.; Larson, D. R.; Webb, W. W. Precise nanometer localization analysis for individual fluorescent probes. Biophys. J. 2002, 82, 2775-2783.

(10) Yildiz, A.; Forkey, J. N.; McKinney, S. A.; Ha, T.; Goldman, Y. E.; Selvin, P. R. Myosin v walks hand-over-hand: single fluorophore imaging with 1.5-nm localization. Science 2003, 300, 2061-2065.

(11) Deschout, H.; Zanacchi, F. C.; Mlodzianoski, M.; Diaspro, A.; Bewersdorf, J.; Hess, S. T.; Braeckmans, K. Precisely and accurately localizing single emitters in fluorescence microscopy. Nature Methods 2014, 11, 253-266.

(12) Small, A.; Stahlheber, S. Fluorophore localization algorithms for super-resolution microscopy. Nature Methods 2014, 11, 267-279.

(13) Heilemann, M.; van de Linde, S.; Schüttpelz, M.; Kasper, R.; Seefeldt, B.; Mukherjee, A.; Tinnefeld, P.; Sauer, M. Subdiffraction-resolution fluorescence imaging with conventional fluorescent probes. Angew. Chem. Int. Ed. 2008, 47, 6172-6176.

(14) Bon, P.; Bourg, N; Lécart, S.; Monneret, S.; Fort, E.; Wenger, J.; Lévêque-Fort, S. Three-dimensional nanometre localization of nanoparticles to enhance super-resolution microscopy. Nat. Commun. 2015, 6, 7764.

(15) Neugebauer, M.; Woźniak, P.; Bag, A.; Leuchs, G.; Banzer, P. Polarization-controlled directional scattering for nanoscopic position sensing. Nat. Commun. 2016, 7, 11286. 
(16) Fernandez-Corbaton, I; Zambrana-Puyalto, X; Tischler, N.; Vidal, X.; Juan, M. L.; Molina-Terriza, G. Electromagnetic duality symmetry and helicity conservation for the macroscopic Maxwell's equations. Phys. Rev. Lett. 2013, 111, 060401.

(17) Tischler, N.; Fernandez-Corbaton, I.; Zambrana-Puyalto, X.; Minovich, A; Vidal, X; Juan, M. L.; Molina-Terriza; G. Experimental control of optical helicity in nanophotonics. Light: Science \& Applications 2014, 3, e183.

(18) Tischler, N.; Juan, M. L.; Singh, S.; Zambrana-Puyalto, X.; Vidal, X; Brennen, G.; Molina-Terriza, G. Topological metrology and its application to optical position sensing. arXiv 2015, $1505.0054 \%$.

(19) Carter, A. R.; King, G. M.; Ulrich, T. A.; Halsey, W.; Alchenberger, D.; Perkins, T. T. Stabilization of an optical microscope to $0.1 \mathrm{~nm}$ in three dimensions. Appl. Opt. 2007, 46, 421-427.

(20) Zambrana-Puyalto, X; Vidal, X; Juan, M. L.; Molina-Terriza, G. Dual and anti-dual modes in dielectric spheres. Optics Express, 2013, 21, 17520-17530.

(21) Person, S.; Jain, M.; Lapin, Z; Sáenz, J. J.; Wicks, G; Novotny, L. Demonstration of Zero Optical Backscattering from Single Nanoparticles. Nano Lett. 2013, 13, 1806-1809.

(22) Züchner, T.; Failla, A. V.; Hartschuh, A.; Meixner, A. J. A novel approach to detect and characterize the scattering patterns of single Au nanoparticles using confocal microscopy. Journal of Microscopy 2008, 229, 337-342.

(23) Balzarotti, F; Eilers, Y.; Gwosch, K. C.; Gynnå, A. H. ; Westphal, V.; Stefani, F. D.; Elf, J.; Hell, S. W. Nanometer resolution imaging and tracking of fluorescent molecules with minimal photon fluxes. Science 2017, 355, 605-612. 


\section{Graphical TOC Entry}

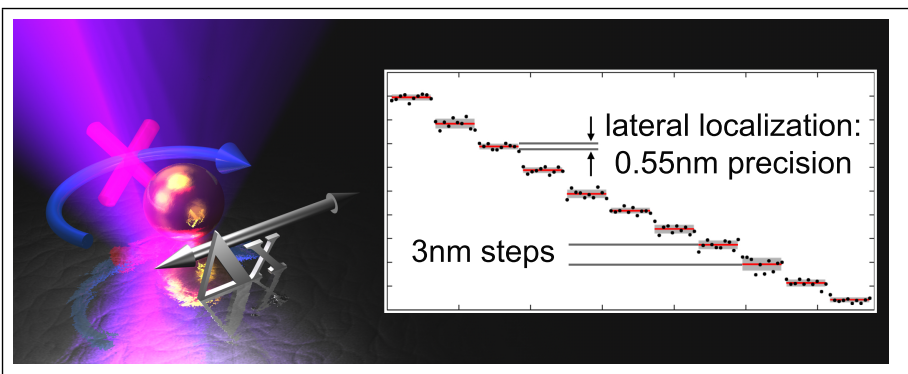

"All-optical self-referenced transverse position sensing with sub-nanometer precision", Nora Tischler, Johannes Stark, Xavier Zambrana-Puyalto, Ivan Fernandez-Corbaton, Xavier Vidal, Gabriel MolinaTerriza, Mathieu L. Juan. 